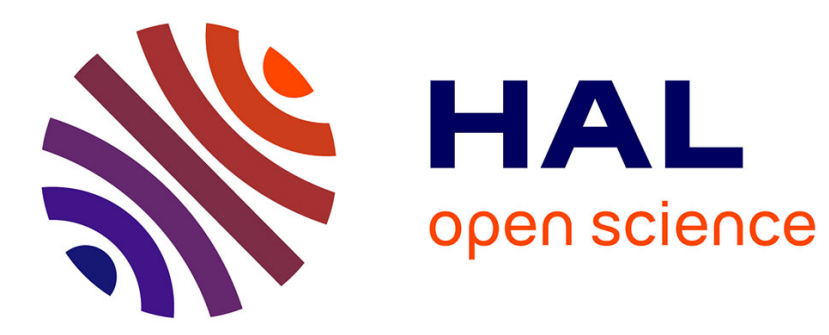

\title{
Spectres de bandes. D'après quelques notes manuscrites de Ritz et une conversation
}

Pierre Weiss

\section{To cite this version:}

Pierre Weiss. Spectres de bandes. D'après quelques notes manuscrites de Ritz et une conversation. Radium (Paris), 1911, 8 (5), pp.177-180. 10.1051/radium:0191100805017700 . jpa-00242468

\section{HAL Id: jpa-00242468 https://hal.science/jpa-00242468}

Submitted on 1 Jan 1911

HAL is a multi-disciplinary open access archive for the deposit and dissemination of scientific research documents, whether they are published or not. The documents may come from teaching and research institutions in France or abroad, or from public or private research centers.
L'archive ouverte pluridisciplinaire HAL, est destinée au dépôt et à la diffusion de documents scientifiques de niveau recherche, publiés ou non, émanant des établissements d'enseignement et de recherche français ou étrangers, des laboratoires publics ou privés. 


\title{
MÉMOIRES ORIGINAUX
}

\author{
Spectres de bandes \\ D'après quelques notes manuscrites de Ritz et une conversation ${ }^{1}$ \\ Par Pierre WEISS
}

\section{a) Mécanisme d'émission des spectres de bandes.}

Dans le mémoire ${ }^{2}$ où Ritz étudie un mécanisme électromagnétique simple émettant les spectres en séries, il dit à la page 675 (OEuvres, p. 112 ).

( Ces théories ne s'appliquent pas aux spectres de bandes. Pour ce qui les concerne, je m'en tiendrai à la remarque que l'on peut peut-être les attribuer ì des anneaux ou à des polygones fermés, formés des mìmes aimants élémentaires. On peut supposer, en effet, que des organes de cette espice jouent un ròle important dans la construction de l'atome et que pour qu'un spectre en séries puisse se produire ils doivent au préalable être brisés par les phénomènes ilectriques ou chimiques qui provoquent l'émission de la lumière.... )

Il a été trouvé dans les papiers laissés par Ritz, sur une petite feuille portant quelques équations et quelques mots de texte, une ébauche de théorie de ce mécanisme d'émission des spectres de bandes, que je vais essayer d'expliciter.

Rappelons quc l'organe d'émission des spectres en séries, imaginé d'abord comme des bâtonnets magnétiques et non magnéliques, juxtaposés en ligne droite, peut être réalisé de diverses facons. Ritz se représentait volontiers ${ }^{5}$ 'es bàtonnets magnétiques comme fournis par des solides de révolution chargés d'électricité à leur surface et animés d'un mouvement rapide de rotation autour de leur axc. Il s'était rendu compte, en particulier, que pour des solides de révolution quelconques on peut trouver une distribution superficielle d'électricité qui les rend équivalents à des systìmes de deux pôles magnétiques situés sur l'axe. Lorsque les pòles magnétiques se rapprochent de la surface, la densité électrique augmente indéfiniment dans leur voisinage et les surfaces portant l'électricité

1. Note ajoutéc aux OEuvres de W. Ritz, publiées par li Soc. Suisse de Phys., (Gauthier-Villars, 1911.)

2. Magnetische Atomfelder und Serienspektren. A $m$. $d$. Phys. 25 (1908) 660; OEuvres de Rit:, VII, p. 98.

3. Ioc. cit. p. 670, OEuvies.

T. 8. deviennent pratiquement équivalentes à des charges ponctuelles. Ces solides sont alternativement positifs et négatifs et doués de rotations de sens contraire. Ils sont fixés les uns aux autres, en chaîne linéaire, par leur attraction électrostatique. Il avait pensé que les bàtonnets non magnétiques, exigés eux aussi par la théorie des spectres en séries, pourraient être des corps électrisés de même espèce, mais dépourvus de rotation, puis, faisant un pas de plus, que l'électron vibrant et le pôle électrique libre à l'extrémité de la file des bâtonnets sont une seule et même chose.

Supposons que, supprimant les bâtonnets non magnétiques, on considère une file de bâtonnets magnétiques. On peut admettre que, lorsqu'elle est soumise à une tension $\alpha^{2}$, elle possède la propriété de prendre des mouvements vibratoires analogues à ceux d'une corde ou plutôt d'une chaine.

Il règne le long de cette corde un champ magnétique $\mathrm{H}$ dirigé dans le sens de la longueur et elle porte des charges électriques équidistantes. Supposons que ces charges exécutent des vibrations circulaires autour de l'axe sous l'influence combinée de la tension $\alpha^{2}$ et du champ H. (Les notes de Ritz sont muettes sur la raison pour laquelle l'action du champ sur les charges positives et négatives voisines ne se neutralisent pas. On peut peut-être invoquer à cet eflet une différence de configuration des charges positives et négatives, que l'on est disposé à admettre par ailleurs. Les unes, occupant un espace plus étendu, seraient, par exemple, partiellement en dehors du champ.)

L'état vibratoire est alors décrit par :

$$
\left\{\begin{array}{l}
y=\mathrm{\Lambda} \sin \frac{m \pi x}{a} \sin v t \\
:=\mathrm{A} \sin \frac{m \pi x}{a} \cos v t
\end{array}\right.
$$

où $a$ est la distance entre deux nouds consécutifs pour la vibration fondamentale et $\nu: 2 \pi$ la fréquence. Les équations du mouvement d'un élément de corde $d x$ de masse $\mu d x$ et de charge $\varepsilon d x$ contiendront le 
terme d'inertie et les forces provenant du champ magnétique et de la tension de la corde :

$$
\left\{\begin{array}{l}
u \frac{\partial^{2} y}{d t^{2}}+\varepsilon H \frac{\partial z}{\partial t}-\jmath^{2} \frac{\partial^{2} y}{\partial x^{2}}=0 \\
u \frac{\partial^{2} z}{\partial t^{2}}-\varepsilon H \frac{\partial y}{\partial t}-\alpha^{2} \frac{\partial^{2} y}{\partial x^{2}}=0
\end{array}\right.
$$

d'où, en substituant (1), deux fois la mème équation :

$$
\mu . \nu^{2}+\varepsilon . \nu . H-\frac{m^{2} \pi^{2}}{a^{2}} \cdot \alpha^{2}=0
$$

dans laquelle $\mathrm{H}$ peut aussi itre remplacé par $-\mathrm{H}$, donc

$$
\text { (4) } \quad \nu= \pm \frac{\varepsilon H}{2 \mu} \pm \frac{\varepsilon H}{2 \mu} \sqrt{1+\frac{4 m^{2} \pi^{2} \mu}{\varepsilon^{2} H^{2} a^{2}} \alpha^{2}} \text {. }
$$

Les solutions correspondant aux deux signes + et aux deux signes - sont seules acceptables. En posant

$$
\frac{4 \pi^{2} \alpha^{2} \mu}{\varepsilon^{2} H^{2} a^{2}}=k^{2}
$$

nombre qui est pelit quand la tension de la corde a un ròle subordonné par rapport au champ, on a :

$$
\text { (5) } \quad v=\frac{\varepsilon \mathrm{H}}{\mu}\left[1+\frac{m^{2} k^{2}}{4}-\frac{m^{4} k^{k}}{16}+\cdots\right] \text {. }
$$

Pour $m=0$, on retrouve la fréquence d'une charge décrivant un cercle dans le champ $\mathrm{H}$. Si l'on s'arrête au deuxième term:, on a la loi de Deslandrıs avec $v_{0}=\frac{\varepsilon H}{\mu}$ pour la tête de la bande. Si l'on conserve le $3^{\text {c }}$ terme, $v$ croît moins vile, comme le veut l'expérience.

Ritz est amené ici à choisir entre une file rectiligne ct un anneau fermé. Il donne la préférence à ce dernier dans les termes suivants : "S'il y a deux extrémités, les lignes devraient être d'abord simples $(m=$ $1,2 \ldots$.$) , puis à m$ grand correspondront des vibrations diverses selon que l'on se trouve aux extrémités ou au milieu. IIonc anncau circulaire »).

$\nu$ croit avec $m$, la bande a donc la tête du cóté du rouge. l'our obtenir des valeurs décroissantes, il faut supposer $x^{2}$ négatif. L'anncau, au lieu d'être tendu, est comprimé dạns la direction de la périphérie.

L'écartement entre deux raies consécutives est donné à partir de la formule complète (4), par :

$$
\frac{d \nu}{d m}=\frac{\nu_{0}}{\omega} \frac{m k^{2}}{\sqrt{1+m^{2} \mathrm{~K}^{2}}}
$$

Il croit plus lentement que la loi de Deslandres l'indique, et cela est conforme à l'expérience. Mais l'écart ne cesse pas de croître. La formule ne donne donc pas le maximum d'écartemeal des expériences de Kayser et Runge ${ }^{1}$ sur le spectre du cyanogène ${ }^{2}$.

1. Kayser et Runge, Wied. Anj., 38 (1889) 80.

2. La même feuille de notes porte encore l'indication sui-
Sur une autre feuille de notes de Ritz se trouve la trace de nombreuses tentatives pour trouver la meilleure formule empirique à trois constantes représentant ces expériences de Kayser et Runge. Cette feuille parait ètre antérieure à ses idées sur l'origine électro-magnétique des spectres et n'est donc qu'indirectement en rapport avec ce qui précède. Il essaye notamment

$$
v=a+b m^{2} \sqrt{1+c m^{2}}
$$

et les 3 premiers termes de son développement

$$
v=a+b m^{2}+c m^{6}
$$

et trouve que le terme en $m^{6}$ varie trop rapidement. Il essaye

et

$$
v^{2}=a+b m^{2}+c m^{4}
$$

$$
v=a+b m^{2}+c m^{4} \text {. }
$$

Il trouve cette dernière formule préférable aux autres et note à ce propos que " à partir de la $160^{\mathrm{e}}$ raie les fonctions $v=f(m)$ et $\nu^{2}=f(m)$ se comportent d'une manière non régulière "). Nous allons revenir sur ce point. Il essaye encore :

$$
\nu^{2}=\frac{a+b m^{2}}{1+c m^{2}}
$$

l'crreur est un peu plus grande que ci-dessus.

Dans une conversation, Ritz avait énoncé une idée qui rattache ce qu'il appelle " lc caractère non régulier de la fonction y pour les raies d'ordre élevé ») au inécanisme d'émission. Il s'était exprimé à peu près de la manière suivante :

On possède dans certaines bandes des nombres de raies considérab!es dont la position est déterminée a vec exactitude; mais, quelle que soit la loi empirique a rec laquelle on cherche à représenter la distribution des raies dans ces bandes, il arrive un moment où, pour un numéro d'ordre élevé des raies, celte loi est en défaut. Si l'on a recours à une représentation graphique, la courbe tourne court, avec une brusquerie que les formules habituelles ne rendent pas.

Imaginons que la partie de l'atome dont les vilurations émeltent des spectres de bandes ait une structure analogue à celle d'une chaine composée de maillons de longueur déterminée. On conçoit alors très bien que les vibrations se produisent pour la plus grande partie du phénomène comme si la chaìne avait une structure continue, tandis que pour des longucurs d'ondes voisines de la longueur du maillon (ou de certaines valeurs particulières en relation arec elle),

vante, se rapportant à une autre solution possible du problème, dans laquelle n'intervient pas la tension $a^{2}:$ "Outre le champ magnétique constant qu'il produit dans ton!e sa longueur, un anneau peut être soumis encore d des champs magnétiques extérieurs, variables de point en point, faibles par rapport au premier ». 
les nombres de vibrations soient influencés par la grandeur finic de l'élément.

\section{b) Structure des bandes.}

A la question qui lui avait été adressée : « N'estil pas établi que des bandes ont quelquefois deux " têtes ", l'une du côté des grandes longueurs d'ondes, l'autre du côté des petites ) (hypothèse de Thiele $\left.{ }^{1}\right)$ ? Ritz avait répondu sans commentaires: "Cette idće n'est pas soutenable ".

Les tableaux de nombres trouvés dans ses notes montrent que cette conviction se rattache aussi à l'élude des bandes du cyanogène observées d'abord par Kayser et Runge ${ }^{2}$, puis par Jungbluth ${ }^{5}$ et dont les têtes sont à $\mathbf{3 8 8 5 , 5 6 ; 3 8 7 1 , 5 5 ; 3 8 6 1 , 8 5}$ et $585 \% 4,85 \AA$.

Voici en quelques mots l'état de la question: $\mathrm{King}^{4}$, ayant découvert de nouvelles têtes dirigées du côté des petites longueurs d'ondes, a cru pouvoir les considérer comme les " queues 》) correspondant aux " tètes " anciennement connues et les a associées en faisant chevaucher les bandes les unes sur les autres. Comme preuve de cette coordination il donne des relations numériques entre les longueurs d'ondes des tètes et des queues. Elles sont contenues dans le tableau suivant:

\begin{tabular}{|c|c|c|c|c|c|}
\hline $\mathrm{T}_{n}$ & $Q_{n}$ & $\frac{\mathrm{T}_{n}}{\mathrm{Q}_{n}}$ & $\mathrm{~T}_{n}$ & $Q_{n}$ & $\frac{\mathrm{T}_{n}}{\mathrm{Q}_{n}}$ \\
\hline 5590,52 & 3203,84 & 1,12069 & 3883,60 & 3465,69 & 1,12059 \\
\hline 5585,99 & 3180,58 & 1,12746 & 3871,59 & 3433,17 & 1,12770 \\
\hline 5584,10 & 5160,32 & 1,13409 & 3861,91 & 3405,04 & $1,13\{17$ \\
\hline
\end{tabular}

La valeur démonstrative de ce tableau me parait faible. D'après la loi de Deslandres, qui est applicable aux tètes de bandes d'une série, comme aux raies d'une bande, les distances entre les têtes successives, mesurées à l'échelle des fréquences, forment une progression arithmétique. Supposons que l'on associe deux séries de bandes tournées en sens contraire et obéissant l'une et l'autre à cette loi, mais entièrement indépendantes quant à leur origine. Si les raisons des deux progressions arithmétiques sont roisines, comme

1. Thiele, Astroph. Journal, 6 (1897) 65.

2. Karser et Ruxge. Wied. Ann., 38 (1889) 80.

3. Juxgbluth. Regularities in the Structure of the third Cyanogen Band. Astroph. Journal, 20 (1904) 237.

4. Kisg. Astroph. Journal, 14 (1901) 323. cela arrive fréquemment', les distances entre les têtes et les queues formeront, elles aussi, une progression arithmétique. (Critérium de dépendance invoqué par Jungbluth.) Il en sera même ainsi, quelles que soient les deux premières bandes que l'on associe, et cela pourra se produire, suivant que les raisons des deux progressions seront de même signe et de signe contraire, quand on se déplace à partir des premières bandes dans le mème sens ou dans le sens contraire.

En première approximation le quotient des fréquences variera, dans les mèmes cas, en progression arithmétique. C'est ce que trouve King. Eu égard ì la grande similitude des séries de bandes successives, on ne saurait attribuer une portée quelconque à ce que ce quotient passe deux fois approximativement par les mêmes trois valeurs.

Cet argument paraît néanmoins avoir pesé d'un assez grand poids dans la conviction de Kayser ${ }^{2}$ qui considère comme certain que King ait trouvé les queues correspondant aux têtes et que par suite l'hypothèse de Thiele soit exacte.

Jungbluth se propose de contrôler cette hypothèse en faisant de nouvelles mesures sur une partie des bandes anciennement connues. Pour les discuter, il porte en abscisses ${ }^{3}$ les longueurs d'ondes et en ordonnées la différence des longueurs d'ondes de deux raies consécutives. Les courbes qu'il obtient ainsi pour quatre des bandes du cyanogène partent de leurs tètes $\mathrm{T}_{1}, \mathrm{~T}_{2}, \mathrm{~T}_{3}, \mathrm{~T}_{4}$ avec une allure approximativement parabolique qui correspond à la loi $v=\mathrm{A}+(\mathrm{B} m+\mathrm{C})^{2}$ ( $\nu=$ fréquence, $m=$ nombre entier) de Deslandres; mais pour les raies de numéros d'ordre élevés, la courbe est nettement au-dessous de la parabole et l'écart entre les raies consécutives passe mème par un maximum. Ces courbes expérimentales sont continuées en pointillé et semblent aboutir naturellement,

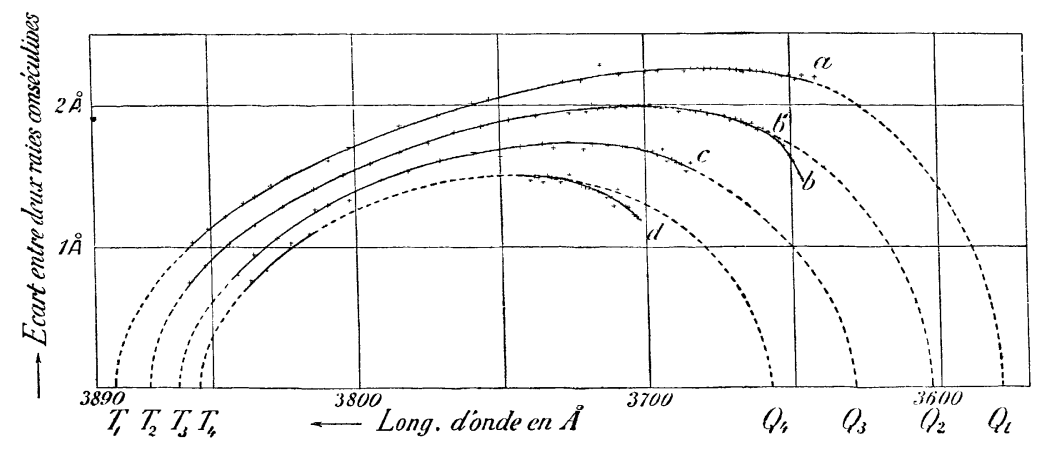

Fig. 1.

dans le dessin original de Jungbluth, pour les trois dernières bandes à des queues $\mathbf{Q}_{2}, Q_{5}, Q_{4}$ indiquées par

1. Voir Fanry. J. de Phys., 4 , 190ذ) 245.

2. Kirser, Handbuch der Spektrost., ire éd. 2-487.

5. La figure donnée ici est celle de Jungbluch, redessinće en remontant aux tableaux de nombres contenus dans son travail. 
King. Pour la première, une tìte de King faisant délaut dans la région où Jungbluth l'attend, il continue la courlıe par continuité, et détermine ainsi la position de la queue $Q_{1}$, daus une région où clle est inobservable par suile de la présence d'une bande intense.

Laissant celle-ci de côté nous avons :

\begin{tabular}{|c|c|c|}
\hline $\begin{array}{c}\text { Tètes } \\
\text { daprès Jungbluth. }\end{array}$ & $\begin{array}{l}\text { Tites } \\
\text { d'après hing. }\end{array}$ & Queucs supposées. \\
\hline $\begin{array}{ll}\mathrm{T}_{2} \ldots . & 5871.55 \\
\mathrm{~T}_{5} \ldots . & 5861,83 \\
\mathrm{~T}_{4} \ldots . & 5854,85\end{array}$ & $\begin{array}{l}4152,93 \\
4158,22 \\
4165,54\end{array}$ & 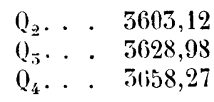 \\
\hline
\end{tabular}

La coordination des têtes et des quenes de Jungbluth est donc formellement en contradiction avec celle de King. En outre, chez Jungbluth. les bandes

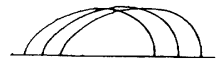

Fig. 2.

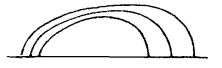

Fig. 5 . complètes de King chevauchant (lig. 2) les unes sur les autres, sont remplacées par des bandes s'embioitont les unes dans les auires.

Jungbluth, qui signale expressément cette circonstance, ne craint pas d'ajouter que, rapprochée des relations numériques de King, ellc apporte aux idées de ce dernice une nouvelle confirmation.

Pour rechercher quelle est exactement la portée des cxpériences de Jungbluth, j’ai marqué dans le dessin les points observés. La partie expérimentale, représentée on traits pleins, se termine, pour les quatre bandes, en $a, b, c, d$. Pour deux des bandes, en $b$ et en $d$, elle s'écarte de la partie extrapolée par Jungbluth, représentée en pointillé, par une plus grande courbure, semblant donner raison à l'idéc de Ritz, et rendint impossible l'altribution des queues faites par Jungbluth.

Ilais si l'on compare les experiences de Junghlulh à celle de Kayser et Runge, la concordance qui est bonne jusqu'en $b^{\prime}$ cesse. On se rend compte facilement que Junghlulh a, en $b^{\prime}$, passé par inadvertance à des raties d'une bande voisine placées par rapport à celles de la bande suivie jusque là à peu prís conme les traits d'un vernier p ir rapport à ceux de l'échelle principale. I'ailleurs Jungbluth supprime dans son graphique la portion $b^{\prime} b$. En définitive sur les quatre bandes, la corrélation entre les tètes et les queues est admissible à la rigueur pour deux d'entre clles $\left(\mathrm{T}_{2}-\mathrm{O}_{2} ; \mathrm{T}_{5}-\mathrm{Q}_{5}\right)$.

L’idée de Ritz sur la nature non régulière de la fonction $v$ pour les raies d'ordre éleré qui repose sur la brusquerie du coude en $b^{\prime}$ a donc en parlie comme origine une erreur de Junglbluth.

Il scmble qu’a la suite du travail de Jungbluth, la conviclion que l'hypothèse de Thicle est exacte se soit généralisée. Lin $1905, \Lambda$. Iagenbach ${ }^{1}$ l'exprime $^{2}$ dans une monographic sur les spectres de bandes. On n'a pas remarqué (qu'en réalité les conclusions de Jungbluth à la fin de son travail sont beaucoup moins affirmatives que ses courbes.

On trouve encore dans les notes de Rit $\iota$ l'indication suivante:

"Les queues de King, en particulier celle à $\mathbf{5 6 0 5}$, sont impossib!es, parce qu'clles sont composées de raies relativement intenses avec des différences presque constantes, tandis que les différences devraient croitre très rapidement comme ì la tête de la bande. )

Cette remarque est très probablement suggérée par l'examen de la planche de Kayser et liunge ${ }^{2}$ sur laquelle il est aisé de reconnaître l'aspect décrit par Ritz. On peut y évaluer la distance des raies à $0, \dot{\jmath} \AA$ environ, ce qui porte l'arc de courbe correspondant bien en dehors des limites de la figure. Le mìne aspect est visible sur la planche de Jungbluth (loc. cil.).

Cet argument semble définitivement délruire ce qu'il reste de probabilité aux attributions faites par Jungbluth. Nous avons déjà fait justice incidemment de l'argument que Jungbluth tire de ce que les longueur's des bandes varient en progression arithmélique. Mentionnons que, par contre, Ritz note arec soin comme un fait important la progression arithmétique, signalée par Jungbluth, des écartements maxima des

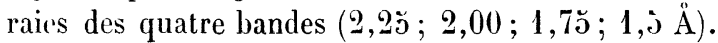
Ce fait conserve sa valeur indépendamment de toute hypothèse sur l'existence d'une seule ou de deux têtes.

On peut donc conclure de la manière suivante: l'hypothìse de Thicle est appuyée par:

$1^{\circ}$ L'existenee des têtes dirig 'es dans les deux sens ;

2o L'existence d'un maximum dans l'écartement des raies.

Mais la démonstration de cetle hypothise n'a j"u ètre continuéc jusquà présent, ni par la possibilité de coordonner sans équiroque les tètes et les queues, ni par la poursuite de la décroissance de la distance des raies dans un intervalle asséz élendu au delà du masinum. L'écartement des ruies dans le voisinağe de la queue $5605 \mathrm{y}$ contredit formellement.

Liidie de Rilz n'est pas en contradiclion arec les faits. Mais les indicalions en sa faveur qui restent dans les quatre bandes du cyanogène sont un peu diminuées après suppression de la partie faulire $b^{\prime} b$ de Jungbluth.

Il y aurait grand intirêt à laire de nouvelles déterminations sur des bandes composées d'un grand nombre de raies et, peut-ètre, à la reprise de la discussion des données déjà connues.

[Nanuscrit reçu le 20 arril 1911].

1. A. ILgenbuch. Wullner-Festschrift (1905) 133.

2. Akad. Bertix. Phys. Abh. nicht zur Aladl. gellör. Gelehrter, 1 (1889) 44. 